\title{
Heart rate variability in juvenile systemic lupus erythematosus patients
}

\author{
Özge Başaran ${ }^{1}$, İbrahim İlker Çetin², Fatma Aydın ${ }^{1}$ Nermin Uncu ${ }^{1}$, Nilgün Çakar ${ }^{3}$, \\ Filiz Ekici², Banu Çelikel Acar ${ }^{1}$ \\ Departments of ${ }^{1}$ Pediatric Rheumatology and ${ }^{2}$ Pediatric Cardiology, University of Health Sciences, Ankara Child Health \\ and Diseases Hematology Oncology Training and Research Hospital; ${ }^{3}$ Department of Pediatric Rheumatology, Ankara \\ University School of Medicine. E-mail: ozgesalor@yahoo.com
}

Received: 30th July 2018, Revised: 27th November 2018, Accepted: 18th February 2019

SUMMARY: Başaran Ö, Çetin İi, Aydın F, Uncu N, Çakar N, Ekici F, Çelikel Acar B. Heart rate variability in juvenile systemic lupus erythematosus patients. Turk J Pediatr 2019; 61: 733-740.

\begin{abstract}
Although neurological involvement has been well recognized in patients with systemic lupus erythematosus (SLE), autonomic nervous system (ANS) involvement has rarely been studied, and has shown conflicting results. The aim of this study was to evaluate the ANS functions by using heart rate variability (HRV) in juvenile patients with SLE. Sixteen juvenile-onset SLE patients and 16 healthy controls were enrolled in the study. All participants underwent 24-hour Holter electrocardiogram monitoring and HRV indices were assessed. The SLE disease activity index (SLEDAI) score was used to assess the disease activity. We analyzed the correlation between disease duration, the SLEDAI score, and the HRV domains. Overall HRV was diminished in patients with SLE compared to controls. There were negative correlations between day and night RMSSD (root-mean-square of the successive normal sinus NN interval differences) and PNN50 (percentage of successive normal sinus $\mathrm{NN}$ intervals $>50 \mathrm{~ms})$ values, and SLEDAI $(\mathrm{r}=-0.588 \mathrm{p}=0.017 ; \mathrm{r}=-0.607$ $\mathrm{p}=0.013 ; \mathrm{r}=-0.498 \mathrm{p}=0.049 ; \mathrm{r}=-0.597 \mathrm{p}=0.015$, respectively). There were positive correlations between both day and night LF/HF values and SLEDAI $(\mathrm{r}=0.766 \mathrm{p}=0.001 ; \mathrm{r}=0.635 \mathrm{p}=0.008$, respectively $)$. The results suggest that autonomic dysfunction exists in juvenile patients with SLE. As these children are at increased risk for cardiovascular disease, they need to be assessed for the development of autonomic dysfunction.
\end{abstract}

Key words: autonomic dysfunction, children, heart rate variability, systemic lupus erythematosus.

Systemic Lupus Erythematosus (SLE) is a connective tissue disease characterized by the formation of autoantibodies and immune complexes. The cardiovascular system involvement is an important manifestation as it is now considered a leading cause of morbidity and mortality in SLE. ${ }^{1}$ Pericardium, myocardium, endocardium, heart valves, coronary vessels, and cardiac autonomic functions may be affected in lupus patients. SLE patients have increase morbidity and mortality due to cardiovascular diseases. ${ }^{2}$ Cardiac autonomic functions could be easily evaluated by non-invasive tests. The heart rate variability (HRV) is one of the best methods that evaluate the relationship between the autonomic nervous system (ANS) and cardiovascular system. HRV has been used as a predictor of cardiovascular disease in several high-risk situations. ${ }^{3}$ Normal heart rate variation depends on the balance between sympathetic and parasympathetic systems. It

This study has previously been presented as a poster presentation in XVII. National Rheumatology Congress, 24-30 October 2016, Antalya, Turkey. 
is defined as the degree of fluctuation of the beat-to-beat differences in cardiac rhythm. Clinical studies have shown that impaired HRV correlates with an increased risk of cardiovascular mortality. ${ }^{4,5}$ It is important to assess the autonomic functions in SLE as some studies proposed that the autonomic fluctuations might predispose to ventricular arrhythmias and circulatory dysfunction. ${ }^{6,7}$ Both sympathetic and parasympathetic pathways are associated with acute and chronic inflammation and autonomic dysfunction in SLE. There are reports suggesting that patients with SLE without clinically apparent cardiac symptoms have impaired autonomic function, primarily in the form of increased sympathetic and decreased parasympathetic activity. ${ }^{3,8}$ On the other hand, in the pediatric population there is only one study that shows the autonomic impairment in SLE up to date. ${ }^{9}$

In this study, we aimed to investigate autonomic system functions in juvenile SLE patients by using HRV.

\section{Material and Methods}

\section{Study Population}

We included 16 juvenile-onset SLE patients (13 female, 3 male, mean age $15.8 \pm 3.2$ years) and 16 healthy controls (13 female, 3 male, mean age $15.1 \pm 2.6$ years) in the study. Diagnosis of SLE was established using the criteria provided by the American College of Rheumatology. ${ }^{10}$ Informed consent was obtained from all subjects; the study was approved by the local ethics committee (number: 2013-045).

Patients were excluded from the study if they had an acute or chronic infection or a chronic disease other than SLE that may affect the ANS. Persons taking any medication that may affect the autonomic nervous system, such as beta-blockers were also excluded. Serum creatinine, acute phase reactants including erythrocyte sedimentation rate (ESR) and c-reactive protein (CRP), complement 3 and 4 levels, auto antibodies including anti-double stranded DNA (anti dsDNA) antibodies and anti nuclear antibodies (ANA) and present medications were obtained from all patients. Treatment with azathioprine, methotrexate, and cyclophosphamide was recorded as immunosuppressive therapy. The activity of SLE was determined according to the Systemic Lupus Erythematosus Disease Activity Index (SLEDAI). ${ }^{11}$

Control subjects were selected from healthy children who did not have any symptoms of ANS dysfunction and were not under any medication.

\section{Cardiac Autonomic Function Evaluation}

We evaluated cardiac autonomic functions by using time-domain and frequency-domain parameters of HRV analysis on 24-hour rhythm Holter electrocardiogram (ECG) recordings. The CardioScan 11.0 software (DM Software Inc., NV, CA, USA) was used to assess HRV. The following parameters were obtained as time-domain measures: Heart rate (minimum, maximum and mean), pulse interval, triangular index (total number of all $\mathrm{NN}$ intervals divided by the height of the histogram of all NN intervals), SDNN (standard deviation of $\mathrm{NN}$ intervals), SDNN index (SD of mean NN intervals of 5-minute records), SDANN (SD of the 5-minute mean NN intervals tabulated over an entire day), RMSSD (root-mean-square of the differences between adjacent NN intervals), and PNN50 (the proportion of differences in successive NN intervals greater than $50 \mathrm{~ms}$ ). The parameters obtained from the frequency-domain analysis of HRV were: Total power, low-frequency band (LF), high-frequency band (HF), and LF/HF ratio. SDANN and SDNN index represent sympathetic activity and reflect diurnal and daytime effects. RMMSD and PNN50 represent direct parasympathetic activity and reflect nighttime effects independently from diurnal effects. Triangular index is a basic parameter for overall HRV and is suppressed by sympathetic effects. LF shows both sympathetic and parasympathetic activity. HF represents parasympathetic activity during nighttime. LF/HF ratio is calculated to exclude parasympathetic components in the LF. While high LF/HF ratios reflect an increase in sympathetic activity, low ratios show an increase in parasympathetic activity.

\section{Statistical Analyses}

All data were processed using the SPSS 
statistical package, version 15 (SPSS, Inc, Chicago, IL, USA). Continuous variables were expressed as mean \pm SD and median (min-max) according to data distribution which was assessed by Kolmogorov-Smirnov test. The Mann-Whitney $U$ test was used for comparison of HRV domains of patients and controls. The Spearman's correlation analysis was used to assess the correlation between the HRV domains and disease activity or disease duration. The $\mathrm{P}$ values below 0.05 were considered significant.

\section{Results}

The age distributions of subjects in the study and control groups were similar $(p=0.296)$. The disease duration of patients ranged from 1 to 150 months (median 38.6 months). Lupus manifestations in this study included systemic features $(100 \%$ cases), renal $(62.5 \%)$, cutaneous $(62.5 \%)$, neurological $(31.25 \%)$, musculoskeletal (25\%), and hematologic (25\%). Patients did not have any concomitant cardiac pathology. Both time-domain and frequency-domain parameters of HRV were compared between the SLE and control groups. The HRV indices obtained from the patients and the controls were given along with the statistical comparison between the groups in Table I. Both time and frequency domain parameters were significantly suppressed in the SLE group than in the control group. Although not statistically significant, LF/HF ratio was higher in the SLE group than in the control group. When we compare the daytime and nighttime periods, the results were as close as the entire day (Table II).

In patients SLEDAI was $10.3 \pm$ 7.9. The correlations between HRV domains and SLEDAI or disease duration were also analyzed. There were negative correlations between day and night RMSSD and PNN50 values, and SLEDAI $(\mathrm{r}=-0.588 \mathrm{p}=0.017$; $r=-0.607 p=0.013 ; r=-0.498 p=0.049 ; r=$ $-0.597 p=0.015$, respectively). There were positive correlations between day and night LF/HF values and SLEDAI $(r=0.766 p=0.001$; $\mathrm{r}=0.635 \mathrm{p}=0.008$, respectively). However, there was no correlation between HRV domains and disease duration. There were also no significant differences in HRV domains
Table I. Baseline Demographic and Laboratory Characteristics of SLE Patients.

\begin{tabular}{|c|c|}
\hline Parameters & Patients $(n=16)$ \\
\hline Age (years) & $15.8 \pm 3.2$ \\
\hline Gender (M/F) & $3 / 13$ \\
\hline Age at diagnosis (years) & $12.81 \pm 3.22$ \\
\hline Disease duration (months) & $38.6(1-150)$ \\
\hline Malar rash & $10(62.5 \%)$ \\
\hline Discoid rash & $1(6.25 \%)$ \\
\hline Photosensitivity & $1(6.25 \%)$ \\
\hline Oral ulcers & $3(18.75 \%)$ \\
\hline Musculoskeletal involvement & $4(25 \%)$ \\
\hline Serositis & $1(6.25 \%)$ \\
\hline Renal involvement & $10(62.5 \%)$ \\
\hline Neurological involvement & $(31.25 \%)$ \\
\hline Hematological involvement & $4(25 \%)$ \\
\hline Antiphospholipid syndrome & $1(6.25 \%)$ \\
\hline Anti-nuclear antibody positivity & $10(62.5 \%)$ \\
\hline Anti-dsDNA positivity & $14(87.5 \%)$ \\
\hline Creatine $(\mathrm{mg} / \mathrm{dl})$ & $0.77 \pm 0.13$ \\
\hline ESR (mm/hour) & $28.31 \pm 20.2$ \\
\hline CRP (mg/dl) (0-0.8) & $0.55 \pm 0.93$ \\
\hline C3 (mg/dl) (83-177) & $86.51 \pm 23.23$ \\
\hline $\mathrm{C} 4(\mathrm{mg} / \mathrm{dl})(12-36)$ & $16.16 \pm 9.08$ \\
\hline Glucocorticoid & $16(100 \%)$ \\
\hline Hydroxychloroquine & $16(100 \%)$ \\
\hline Immunosuppressive agents & $10(62.5 \%)$ \\
\hline
\end{tabular}

Data are given as number (\%) or mean $\pm \mathrm{SD}$. antidsDNA, anti-double stranded DNA antibodies; F, female; $\mathrm{M}$, male; ESR, erythrocyte sedimentation rate; CRP, C-reactive protein.

between patients having central nervous system (CNS) involvement and not $(\mathrm{p}=0.420$ for $L F, p=0.263$ for HF, $p=0.950$ for SDNN, $\mathrm{p}=0.093$ for SDNNI, $\mathrm{p}=0.562$ for SDANN, and $\mathrm{p}=0.147$ for $\mathrm{pNN} 50$ ).

\section{Discussion}

This study has revealed that the time and frequency-domain HRV parameters are impaired in children with SLE, which can represent the presence of sympathovagal imbalance. 
Table II. Comparison of Heart Rate Variability Parameters Between Patients with SLE and Healthy Control.

\begin{tabular}{|c|c|c|c|}
\hline HRV parameters & $\begin{array}{c}\text { Juvenile SLE patients } \\
\text { Mean } \pm \text { SD } \\
\text { Median (min-max) }\end{array}$ & $\begin{array}{c}\text { Controls } \\
\text { Mean } \pm \text { SD } \\
\text { Median (min-max) }\end{array}$ & $\mathrm{p}$ \\
\hline \multirow{2}{*}{ Min. heart rate (beats/min) } & $50 \pm 6$ & $47 \pm 3$ & \multirow{2}{*}{ NS } \\
\hline & $50(40-66)$ & $47(40-53)$ & \\
\hline \multirow{2}{*}{ Max. heart rate (beats/min) } & $160 \pm 23$ & $162 \pm 17$ & \multirow{2}{*}{ NS } \\
\hline & $163(123-200)$ & $164(126-188)$ & \\
\hline \multirow{2}{*}{ Mean heart rate (beats/min) } & $88 \pm 8$ & $79 \pm 7$ & \multirow{2}{*}{$<0.01$} \\
\hline & $89(77-106)$ & $79(69-89)$ & \\
\hline \multirow{2}{*}{ Pulse interval } & $111 \pm 23$ & $115 \pm 18$ & \multirow{2}{*}{ NS } \\
\hline & $111(77-150)$ & $117(79-148)$ & \\
\hline \multirow{2}{*}{ Total Power (ms2) } & $3201 \pm 1372$ & $5830 \pm 2079$ & \multirow{2}{*}{$<0.001$} \\
\hline & $3399(986-5511)$ & $5561(3201-10814)$ & \\
\hline \multirow{2}{*}{$\mathrm{LF}(\mathrm{ms} 2)$} & $718 \pm 313$ & $1218 \pm 375$ & \multirow{2}{*}{$<0.001$} \\
\hline & $732(236-1222)$ & $1182(579-2031)$ & \\
\hline \multirow{2}{*}{$\mathrm{HF}(\mathrm{ms} 2)$} & $395 \pm 218$ & $693 \pm 271$ & \multirow{2}{*}{$<0.01$} \\
\hline & $392(53-834)$ & $639(364-1472)$ & \\
\hline \multirow{2}{*}{ LF/HF } & $2,2 \pm 1,3$ & $1,9 \pm 0,7$ & \multirow{2}{*}{ NS } \\
\hline & $1,9(1,2-6,1)$ & $1,9(1,1-3,3)$ & \\
\hline \multirow{2}{*}{ Triangular index } & $36 \pm 12$ & $51 \pm 13$ & \multirow{2}{*}{$<0.01$} \\
\hline & $32(20-67)$ & $49(31-77)$ & \\
\hline \multirow{2}{*}{ SDNN (ms) } & $129 \pm 32$ & $158 \pm 34$ & \multirow{2}{*}{$<0.05$} \\
\hline & $136(68-193)$ & $152(111-218)$ & \\
\hline \multirow{2}{*}{ SDNN index (ms) } & $56 \pm 13$ & $78 \pm 14$ & \multirow{2}{*}{$<0.01$} \\
\hline & $60(30-77)$ & $75(58-114)$ & \\
\hline \multirow{2}{*}{ SDANN (ms) } & $121 \pm 32$ & $134 \pm 35$ & \multirow{2}{*}{ NS } \\
\hline & $128(62-174)$ & $130(86-201)$ & \\
\hline \multirow{2}{*}{ RMSSD (ms) } & $37 \pm 14$ & $53 \pm 15$ & \multirow{2}{*}{$<0.01$} \\
\hline & $37(13-69)$ & $54(34-100)$ & \\
\hline \multirow{2}{*}{ PNN50 (\%) } & $15 \pm 10$ & $26 \pm 9$ & \multirow{2}{*}{$<0.01$} \\
\hline & $13(0-39)$ & $24(13-49)$ & \\
\hline \multirow{2}{*}{ Daytime Total Power (ms2) } & $2903 \pm 1435$ & $5392 \pm 2066$ & $<0.001$ \\
\hline & $2922(854-5567)$ & $5200(3006-10623)$ & \\
\hline Davtime LF (ms2) & $644 \pm 339$ & $1097 \pm 325$ & $<0.001$ \\
\hline & $602(199-1340)$ & $1043(556-1655)$ & \\
\hline Davtime HF (ms2) & $282 \pm 214$ & $545 \pm 229$ & $<0.01$ \\
\hline Dof & $258(34-930)$ & $508(285-1193)$ & -0.01 \\
\hline Doutime I F/HF & $2,9 \pm 1,7$ & $2,2 \pm 0,8$ & NS \\
\hline 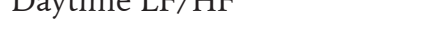 & $2,4(1,4-6,9)$ & $2,1(1,1-3,5)$ & 1No \\
\hline
\end{tabular}


Table II. Continue.

\begin{tabular}{|c|c|c|c|}
\hline HRV parameters & $\begin{array}{c}\text { Juvenile SLE patients } \\
\text { Mean } \pm \text { SD } \\
\text { Median (min-max) }\end{array}$ & $\begin{array}{c}\text { Controls } \\
\text { Mean } \pm \text { SD } \\
\text { Median (min-max) }\end{array}$ & $\mathrm{p}$ \\
\hline \multirow{2}{*}{ Daytime SDNN (ms) } & $114 \pm 27$ & $140 \pm 32$ & \multirow{2}{*}{$<0.05$} \\
\hline & $116(70-165)$ & $136(83-206)$ & \\
\hline \multirow{2}{*}{ Daytime RMSSD (ms) } & $32 \pm 14$ & $47 \pm 14$ & \multirow{2}{*}{$<0.01$} \\
\hline & $30(11-71)$ & $45(30-90)$ & \\
\hline \multirow{2}{*}{ Daytime pNN50 (\%) } & $10 \pm 9$ & $21 \pm 9$ & \multirow{2}{*}{$<0.01$} \\
\hline & $9(0-37)$ & $17(9-42)$ & \\
\hline \multirow{2}{*}{ Night Total Power (ms2) } & $3885 \pm 1729$ & $6670 \pm 2676$ & \multirow{2}{*}{$<0.01$} \\
\hline & 3757 (1301-7041) & $5862(3568-14084)$ & \\
\hline \multirow{2}{*}{ Night LF (ms2) } & $886 \pm 464$ & $1431 \pm 677$ & \multirow{2}{*}{$<0.05$} \\
\hline & 745 (321-2079) & $1277(673-3222)$ & \\
\hline \multirow{2}{*}{ Night HF (ms2) } & $637 \pm 360$ & $970 \pm 453$ & \multirow{2}{*}{$<0.05$} \\
\hline & $672(72-1381)$ & $822(477-1934)$ & \\
\hline \multirow{2}{*}{ Night LF/HF } & $1,9 \pm 1,6$ & $1,7 \pm 0,8$ & \multirow{2}{*}{ NS } \\
\hline & $1,5(0,7-7,2)$ & $1,6(0,6-3,5)$ & \\
\hline \multirow{2}{*}{ Night SDNN (ms) } & $95 \pm 28$ & $122 \pm 26$ & \multirow{2}{*}{$<0.01$} \\
\hline & $95(51-139)$ & $113(87-171)$ & \\
\hline \multirow{2}{*}{ Night RMSSD (ms) } & $49 \pm 18$ & $65 \pm 19$ & \multirow{2}{*}{$<0.05$} \\
\hline & $50(17-79)$ & $62(42-119)$ & \\
\hline \multirow{2}{*}{ Night PNN50 (\%) } & $27 \pm 16$ & $38 \pm 11$ & \multirow{2}{*}{$<0.05$} \\
\hline & $26(1-52)$ & $38(21-63)$ & \\
\hline
\end{tabular}

Data are given as mean \pm SD or median (minimum-maximum)

Triangular index, total number of all NN intervals divided by the height of the histogram of all NN intervals; SDNN, standard deviation of NN intervals; SDNN index, SD of mean NN intervals of 5-minute records; SDANN, SD of the 5 -minute mean NN intervals tabulated over an entire day; RMSSD, square root of the mean of the sum of the squares of differences between adjacent NN intervals; pNN50, the proportion of differences in successive NN intervals greater than $50 \mathrm{~ms}$; LF, low-frequency band; HF, high frequency band.

In SLE, ANS dysfunction was seen in 6-93\% of cases. The pathogenesis of ANS dysfunction in SLE patients still remains unclear. An immunological mechanism may have a role in the pathogenesis. Some studies found no relationship between autoantibody positivity and the ANS dysfunction while others found a significant negative correlation. ${ }^{2,12,13}$ In addition to this, neuropathy and the ANS dysfunction in SLE also arise from vasculitis and drug toxicity. In their study of the role of glucocorticoids in autonomic neuropathy, Liote et al. ${ }^{14}$ revealed that autonomic neuropathy could be related to steroid use in SLE. Peripheral nervous system involvement is also seen in $2-18 \%$ of adult SLE patients. Liote et al. ${ }^{14}$ and Shalimar et al. ${ }^{15}$ did not show a relation between peripheral neuropathy and autonomic neuropathy in their cohorts. None of the patients in our study had clinical and symptomatic peripheral neuropathy. Therefore, peripheral and autonomic neuropathy could not be compared in this study.

The ANS can be evaluated in several ways including cardiovascular, sweating, pupillary reflex, skin, and pharmacological tests. ${ }^{16}$ The HRV is a non-invasive way and results are easy to reproduce. Moreover, the HRV measurement is one of the best methods that 
evaluate the relationship between ANS and cardiovascular system quantitatively. ${ }^{6}$ The HRV is defined as beat-to-beat variability in heart rate. The leading cause of HRV is autonomic tonus alterations. The variability in heart rate is physiological and an expected condition in healthy people. Central and peripheral mechanisms can both contribute to beat-tobeat changes in heart rate. Highly variable heart rates are a sign of good autonomic control. ${ }^{4-6}$ The HRV is assessed by time and frequencydomain variables. There is a general agreement that the high-frequency band (HF) reflects parasympathetic activity and low-frequency band (LF) reflects both parasympathetic and sympathetic activity. The LF/HF ratio is an indicator of sympathovagal balance. ${ }^{17}$ The SDNN represents the overall HRV and the SDANN provides long-term information about HRV. The RMSSD and PNN50 values are used as an indicator of parasympathetic activity. Increments of those two parameters represent a parasympathetic hyperactivity and loss of sympathetic activity. ${ }^{17-19}$ It has been reported that low values of these parameters due to parasympathetic dysfunction precede increased inflammatory activity and raise cardiovascular mortality and morbidity. ${ }^{20}$

Non-invasive cardiovascular reflex tests have been used in some studies to evaluate cardiovascular ANS in rheumatologic disorders. They found significantly diminished heart rate response in RA and SLE patients. ${ }^{2,8,12,15,21}$ In current study, we only used HRV parameters to show the autonomic dysfunction in juvenile lupus patients. Similar to our study some authors used HRV to evaluate the autonomic dysfunctions. Milovanovic et al. ${ }^{22}$ used both HRV test and cardiac reflex test together and reported that the HRV analysis is a more sensitive method than a cardiovascular reflex test to show the subclinical forms of autonomic dysfunction. They concluded that the patients with RA and SLE have marked autonomic dysfunction with sympathetic predominance in the patients with SLE and parasympathetic predominance in the patients with RA. Poliwczak et al. ${ }^{23}$ reported heart rate turbulence and HRV results of their adult patients with SLE without apparent heart disease. Their study confirmed the presence of autonomic disorders with respect to both
HRV and heart rate turbulence parameters and the presence of diurnal disturbances of sympathetic-parasympathetic balance. Thanou et al. ${ }^{3}$ found potential relationships between HRV domains and disease activity measured by SLEDAI and BILAG (British Isles Lupus Assessment Group). They also reported correlated HRV changes (time-domain analysis and HF power) with disease flares. They found an inverse correlation between HRV domains and SLEDAI. In our study, we found inverse correlations in RMSSD and PNN50, but we found positive correlation between LF/HF ratio and SLEDAI. The SLEDAI in our study $(10.3 \pm 7.9)$ was high. Therefore, the HRV results and the direction of their correlation with the SLEDAI might be attributed to higher SLEDAI scores of patients in the current study.

In the literature, there has been only one pediatric data regarding cardiac autonomic functions in patients with rheumatic diseases to compare our results. El Sayed et al. ${ }^{9}$ assessed cardiovascular autonomic function in 20 lupus and 20 juvenile idiopathic arthritis (JIA) patients by using five noninvasive autonomic function tests including HRV and serum levels of neuropeptide $\mathrm{Y}$ and vasoactive intestinal peptide and compared with 40 matched healthy controls. It was found that autonomic function test results had a significantly positive correlation with important disease manifestations, including activity. Thus, it was concluded that cardiovascular autonomic neuropathy might play a role in the pathophysiology of those autoimmune diseases. They also mentioned that cardiovascular autonomic neuropathy is a common occurrence in lupus and JIA patients, even in the absence of relevant symptoms and recommended assessment of autonomic function in these patients.

There are some limitations of the current study that need to be mentioned. First of all, the number of patients included in the study is small and the sample may not represent overall juvenile SLE patients. The second limitation is the cross-sectional nature of the study. Since HRV was evaluated in any period of the disease in this study it is not possible to know the effects of immune suppression therapies and the disease severity on HRV or vice versa. But we think that a randomized 
controlled trial in children should be done to qualify the importance of immune suppressive therapies on HRV in autoimmune disorders. In addition, only one method was used to analyze the autonomic dysfunction so we could not compare the other non-invasive autonomic reflex tests.

In conclusion, significantly diminished HRV domains were found in juvenile SLE patients when compared to the control group in this study. These results indicate that autonomic functions may be impaired in juvenile SLE patients. In addition, autonomic dysfunction may be affected by the disease activity. As patients with SLE are at increased risk for developing cardiovascular disease, they need to be followed closely for autonomic dysfunction. Large-scale, prospective, and randomized controlled studies are needed for further evaluation of cardiac autonomic function assessment in juvenile SLE patients.

\section{REFERENCES}

1. Kojuri J, Nazarinia MA, Ghahartars M, Mahmoody Y, Rezaian Gr, Liaghat L. QT dispersion in patients with systemic lupus erythematosus: the impact of disease activity. BMC Cardiovasc Disord 2012; 12: 11.

2. Aydemir M, Yazisiz V, Basarici I et al. Cardiac autonomic profile in rheumatoid arthritis and systemic lupus erythematosus. Lupus 2010; 19: 255261.

3. Thanou A, Stavrakis S, Dyer JW, Munroe ME, James JA, Merrill JT. Impact of heart rate variability, a marker for cardiac health, on lupus disease activity. Arthritis Res Ther 2016; 18: 197.

4. Kolsal E, Serdaroğlu A, Cilsal E, et al. Can heart rate variability in children with epilepsy be used to predict seizures? Seizure 2014; 23: 357-362.

5. Kilic A, Gulgun M, Tascilar ME, Sari E, Yokusoglu M. Cardiac autonomic regulation is disturbed in children with euthyroid Hashimoto thyroiditis. Tohoku J Exp Med 2012; 226: 191-195.

6. Kaya H, Süner A, Köroğlu S, Akçay A, Türkbeyler İH, Köleoğlu M. Heart rate variability in familial Mediterranean fever patients. Eur J Rheumatol 2014; 1: $58-61$

7. De Ferrari GM, Schwartz PJ. Autonomic nervous system and arrhythmias. Ann N Y Acad Sci 1990; 601: 247-262.

8. Yorgun H, Canpolat U, Aytemir K, et al. Evaluation of cardiac autonomic functions in patients with systemic lupus erythematosus. Lupus 2012; 21: 373-379.
9. El-Sayed ZA, Mostafa GA, Aly GS, El-Shahed GS, Abd El-Aziz MM, El-Emam SM. Cardiovascular autonomic function assessed by autonomic function tests and serum autonomic neuropeptides in Egyptian children and adolescents with rheumatic diseases. Rheumatology (Oxford) 2009; 48: 843-848.

10. Tan EM, Cohen AS, Fries JF, et al. The 1982 revised criteria for the classification of systemic lupus erythematosus. Arthritis Rheum 1982; 25: 12711277.

11. Bombardier C, Gladman DD, Urowitz MB, Caron $\mathrm{D}$, Chang $\mathrm{CH}$. Derivation of the SLEDAI. A disease activity index for lupus patients. The Committee on Prognosis Studies in SLE. Arthritis Rheum 1992; 35: 630-640.

12. Altomonte L, Mirone L, Zoli A, Magaro M. Autonomic nerve dysfunction in systemic lupus erythematosus: evidence for a mild involvement. Lupus 1997; 6: 441444.

13. Baerwald C, Graefe C, Muhl C, Von Wichert P, Krause A. Beta 2-adrenergic receptors on peripheral blood mononuclear cells in patients with rheumatic diseases. Eur J Clin Invest 1992; 22 (Suppl 1): 42-46.

14. Lioté F, Osterland CK. Autonomic neuropathy in systemic lupus erythematosus: cardiovascular autonomic function assessment. Ann Rheum Dis 1994; 53: 671-674.

15. Shalimar, Handa R, Deepak KK, Bhatia M, Aggarwal P, Pandey RM. Autonomic dysfunction in systemic lupus erythematosus. Rheumatol Int 2006; 26: 837840.

16. Louthrenoo W, Ruttanaumpawan P, Aramrattana A, Sukitawut W. Cardiovascular autonomic nervous system dysfunction in patients with rheumatoid arthritis and systemic lupus erythematosus. QJM 1999; 92: 97-102.

17. Özdemir R, Olukman Ö, Karadeniz C, et al. Effect of unconjugated hyperbilirubinemia on neonatal autonomic functions: evaluation by heart rate variability. J Matern Fetal Neonatal Med 2018; 31: 2763-2769.

18. Kardelen F, Tezcan G, Akcurin G, Ertug H, Yesilipek A. Heart rate variability in patients with thalassemia major. Pediatr Cardiol 2008; 29: 935-939.

19. Verklan MT, Padhye NS. Spectral analysis of heart rate variability: an emerging tool for assessing stability during transition to extrauterine life. J Obstet Gynecol Neonatal Nurs 2004; 33: 256-265.

20. Jarczok MN, Kleber ME, Koenig J, et al. Investigating the associations of self-rated health: heart rate variability is more strongly associated than inflammatory and other frequently used biomarkers in a cross sectional occupational sample. PLoS One 2015; 10: e0117196. 
21. Stojanovich L, Milovanovich B, de Luka SR, et al. Cardiovascular autonomic dysfunction in systemic lupus, rheumatoid arthritis, primary Sjögren syndrome and other autoimmune diseases. Lupus 2007; 16: 181-185.

22. Milovanović B, Stojanović L, Milićevik N, Vasić K, Bjelaković B, Krotin M. Cardiac autonomic dysfunction in patients with systemic lupus, rheumatoid arthritis and sudden death risk. Srp Arh Celok Lek 2010; 138: 26-32.

23. Poliwczak AR, Waszczykowska E, DziankowskaBartkowiak B, Koziróg M, Dworniak K. The use of heart rate turbulence and heart rate variability in the assessment of autonomic regulation and circadian rhythm in patients with systemic lupus erythematosus without apparent heart disease. Lupus 2018; 27: 436-444. 